\title{
Procedure End Reference Time Point
}

National Cancer Institute

\section{Source}

National Cancer Institute. Procedure End Reference Time Point. NCI Thesaurus. Code C117542.

A point in time that indicates the conclusion of a procedure. 